\title{
¿CÓMO SE ASIMILAN LAS PREPOSICIONES PROPIAS ITALIANAS? ACERCAMIENTO A LA CUESTIÓN A PARTIR DEL ANÁLISIS DEL OUTPUT DE APRENDICES HISPANOHABLANTES DE ITALIANO/LE EN LA PRIMERA FASE DE SU INTERLENGUA
}

\author{
Carmen Solsona Martinez.
}

Universidad de Zaragoza

\begin{abstract}
RESUMEN
El objetivo de nuestra contribución es presentar un análisis del caudal lingüístico de un grupo de aprendices hispanohablantes de italiano/LE en la primera fase de su interlengua y en una prueba de producción escrita, centrándonos en el uso que hacen de las llamadas preposiciones propias del italiano. Para llevar a cabo el estudio, nos hemos basado en los métodos del Análisis de Errores y de la Interlengua, teniendo también presentes las observaciones del Análisis Contrastivo. Recogimos todas las frases en las que aparecía una preposición y también aquéllas en las que debería haber aparecido. Se analizaron los usos correctos, los usos incorrectos, las preposiciones más usadas y con qué valores, las preposiciones menos usadas, las nunca usadas, las preposiciones que ofrecian más dificultad, los errores cometidos en el empleo de cada preposición, los complementos que presentaban mayor porcentaje de error y las estrategias empleadas por los aprendices cuando carecen todavía de recursos en la lengua meta. De los resultados se extraen algunas conclusiones que pueden ser de utilidad para la práctica docente.
\end{abstract}

Palabras clave: preposiciones propias italianas, aprendices hispanohablantes de italiano/LE, análisis del output.

ABSTRACT

The objective of our contribution is to present an analysis of the output of a group of Spanish-speaking learners of Italian as a foreign language in the first phase of their interlanguage and in a test of written production, in which we focus on the use they make of the so-called proper prepositions of Italian. To carry out this study, we have based it on Error Analysis and Interlanguage Analysis methods, and we have likewise considered the observations of the Contrastive Analysis. We included all the sentences in which a preposition appeared, and also those in which a preposition should have appeared. An analysis was made of the correct uses, the incorrect uses, the most-used prepositions and their values, the least-used prepositions, those never used, the prepositions that presented the most difficulty, the errors made when using each preposition, the complements that showed the greatest error percentage and the strategies used by the learners when they still lacked resources in the target language. Some conclusions are reached, which can be useful for the teaching practice. 
Keywords: Proper prepositions of Italian, Spanish-speaking learners of Italian/FL, output analysis.

\section{OBJETO DE LA INVESTIGACIÓN}

El objetivo de nuestra contribución es presentar un análisis del caudal lingüístico ${ }^{1}$ producido por un grupo de 57 aprendices hispanohablantes de italiano/LE en la primera fase de su interlengua ${ }^{2}$ y en una prueba de producción escrita, centrándonos en el uso que hacen de las llamadas 'preposiciones propias' del italiano.

Otros años, los alumnos de primer nivel comenzaban a realizar composiciones escritas cuando se habían explicado en clase y se habían puesto suficientemente en práctica determinados elementos gramaticales que suponíamos indispensables para poder realizar una composición de aproximadamente 80-100 palabras: artículos determinados e indeterminados, presente de indicativo de verbos regulares e irregulares, género y número, determinantes posesivos, preposiciones y un vocabulario aproximado de 600 palabras. Pues bien, queríamos que esta prueba nos sirviese para ver qué preposiciones y qué valores de esas preposiciones usaba el aprendiz y cómo las empleaba sin haber explicado este tema gramatical en clase (sin decir siquiera cuántas preposiciones hay en italiano, ni los valores que tienen, ni que se contraen con el artículo determinado formando las preposiciones articuladas, ni que se omiten o aparecen en contextos distintos a los del español), sino habiéndolas trabajado, en todo caso, de forma inductiva (el profesor en clase habla en italiano y las usa obviamente,

\footnotetext{
${ }^{1}$ La realidad nos dice que los aprendices de una lengua no asimilan toda la información lingüística a la que están expuestos (input) y también que las reglas y construcciones de sus interlenguas (output) no siempre proceden del input al que han estado expuestos. Quien en definitiva controla el input es el aprendiz que, al asimilarlo, lo convierte en intake. Así pues, el input es todo el material lingüístico que el aprendiz tiene a su disposición (oral y escrito); el intake lo forman los datos lingüísticos que el aprendiz asimila y está condicionado por muy distintos factores (conocimiento lingüístico previo que el aprendiz posee de la L2, de la L1 y de otras lenguas, capacidad para resolver problemas, para hacer inferencias, analogías, comparaciones, etc.), y el output es todo el caudal lingüístico producido por el aprendiz, es decir, su interlengua.

${ }^{2}$ Con el término interlengua -en la bibliografía de enseñanza-aprendizaje de una L2- se hace referencia tanto al tipo de lengua producido por quien aprende una L2 o una LE en una determinada fase de su proceso de aprendizaje, como al conjunto de sistemas lingüísticos interconectados que caracterizan el proceso de aprendizaje de un alumno a lo largo del tiempo (con sus estrategias, sus aciertos que serán cada vez mayores, y sus errores que irán disminuyendo conforme el alumno alcance niveles de competencia mayores). En nuestro estudio nos referimos a la primera acepción, puesto que el análisis se limita a la primera fase del proceso de aprendizaje de la L2 (en el contexto de aprendizaje de aula en el que se lleva a cabo el estudio, los alumnos han realizado la prueba cuando habían recibido 35 horas de clase).
} 
aparecen en los ejercicios y en los textos propuestos, y las han usado -sin ser corregidos más que en contadas ocasiones- en la conversación diaria en clase).

¿Para qué? Para analizar la manera que tienen los alumnos de procesar el input de la L2 (averiguando qué preposiciones -de las ocho preposiciones propias italianas: $a$, con, da, di, in, per, su, tra/fra- se asimilan antes y mejor y, en concreto, en relación con la composición sometida a análisis, qué valores de cada preposición son los que aprenden primero y cuáles no aparecen nunca, ver si -como afirma la psicología cognitiva- perciben en primer lugar la noción del espacio, en segundo lugar la del tiempo y en tercer lugar el resto de las nociones 3 ), para estudiar qué estrategias ponen en funcionamiento los alumnos cuando les faltan recursos en la L2, para comprobar en qué medida es necesaria la instrucción de determinados elementos lingǘsticos y si el número de preposiciones por número de palabras aumenta con la instrucción o se mantiene más o menos invariable ${ }^{4}$. Está claro que alguna decisión tienen que adoptar los alumnos, porque no van a dejar espacios en blanco en lugar de preposiciones por no haberlas estudiado ya que, además, las preposiciones son elementos lingüísticos indispensables para conectar palabras y para construir muchas expresiones de tiempo, lugar, modo, etc. que les iban a hacer falta.

\section{SuJETOS DE LA PRUEBA}

Para poder llevar a cabo el estudio, pedimos a nuestros alumnos de primer curso de italiano/L2 (alumnos universitarios de primer curso de Filología Hispánica, Inglesa, Francesa y de otras facultades, principalmente Derecho y Geológicas) que escribiesen una composición con el título "Mi presento" de una extensión aproximada de 80-150 palabras. De los 70 alumnos matriculados, realizaron la composición 57 alumnos. Se trató de una prueba de carácter voluntario que se sugirió en diciembre como tarea de deberes para casa que debían entregar cinco días más tarde. En el momento de realizar la prueba, los alumnos habían recibido 35 horas de clase, en las que se habían visto los siguientes contenidos gramaticales: artículos determinados e indeterminados, presente de indicativo de verbos regulares e irregulares, género y número, posesivos y demostrativos. También se había revisado el léxico correspondiente a campos semánticos como: las nacionalidades, el vocabulario relativo a presentaciones, colores, tiendas, prendas de ropa, objetos del aula, alimentos y bebidas, localizaciones en la ciudad, regiones y ciudades de Italia. Por último, las funciones comunicativas

\footnotetext{
3 Así es como C. Fernández López (1999) presenta los distintos valores de las preposiciones (empezando por el espacio, luego el tiempo y, a continuación, el resto de las nociones).

${ }^{4}$ Este ejercicio constituye una de las pruebas de una investigación más amplia llevada a cabo durante el año académico 2002-2003 con un grupo de 57 aprendices españoles de italiano como L2. En ella hemos querido analizar la interlengua de los aprendices, centrándonos en el modo que tienen de adquirir las preposiciones propias italianas, las estrategias de aprendizaje utilizadas, los errores más frecuentes y sus posibles causas.
} 
presentadas habían sido: saludar, presentarse, pedir permiso, agradecer, solicitar y ofrecer una información ${ }^{5}$.

\section{MÉTOdo}

Para nuestro estudio nos hemos basado en los métodos del Análisis de Errores (AE) y de la Interlengua (IL), teniendo también en cuenta las observaciones del Análisis Contrastivo (AC). Por eso, hablamos de una L1 o lengua materna del aprendiz (español en nuestro caso), de una L2 o LE (italiano), y lo que realmente es nuestro objeto de estudio: un sistema aproximado (o interlengua) que no se corresponde con la norma correcta estándar de la L2, pero refleja la competencia que de ella tiene el aprendiz en ese momento y que, en algunos casos, incluye rasgos de su sistema nativo.

La extensión media de las composiciones ha sido de 120 palabras. Hemos recogido todas las frases en las que aparecía una preposición y también aquéllas en las que debería haber aparecido. Se han analizado: los usos correctos, los usos incorrectos, las preposiciones más usadas y con qué valores, las preposiciones menos usadas, las nunca usadas, las preposiciones que han ofrecido más dificultad, los errores cometidos en cada preposición y los complementos que han presentado mayor porcentaje de error. También hemos tratado de valorar la posible práctica de estrategias de evitación o inbibición (avoidance) por parte de los estudiantes. No se han tenido en cuenta los errores concernientes a otros aspectos que no tengan que ver con las preposiciones (sí, en cambio, los que guardan relación con ellas, como la contracción de preposición y artículo determinado).

La preposición representa en las composiciones el 9\% del total de palabras escritas. Antes de comentar los resultados, nos gustaría hacer alguna precisión. Al ir anotando los usos correctos e incorrectos en cada una de las composiciones, nos encontramos con el problema de valorar y, en qué medida, los errores cometidos en el campo de los artículos determinados cuando aparecían junto con la preposición. Sabemos que las preposiciones $a, d i, d a, i n$, su se contraen con el artículo determinado formando las preposiciones articuladas, mientras que con per, con, tra/fra no tiene lugar la contracción.

Nos hemos encontrado con más contracciones de las que esperábamos. Pensábamos que, puesto que es un aspecto particular de la gramática italiana en el campo preposicional que no habíamos tratado explícitamente, los alumnos no iban a contraer, o - como mucho - en aquellos casos en los que lo hace el español ( $a l$, del). Los resultados han revelado lo contrario: hay bastantes casos de contracción, aunque

\footnotetext{
${ }^{5}$ Se les dijo que, para realizar la composición, utilizasen el vocabulario y las expresiones vistas en clase y que podían utilizar el diccionario si lo consideraban oportuno. El tema "Mi presento" les permitía escribir de cosas que se habían practicado en clase de forma oral: cómo se llamaban, dónde habían nacido, cuáles eran sus aficiones, cómo eran sus familias, qué estudiaban, etc.
} 
no hayan dado soluciones completamente correctas. Por tanto, si contábamos como incorrecta una contracción no realizada (mi alio a le sette, ejemplo 1$)^{6}$ y un acierto ( $m i$ alzzo alle sette); ¿qué hacíamos con casos como mi alžo alla sette (ejemplo 3) o mi piace parlare con miei amici (ejemplo 5)? En el ejemplo 5, se ve con claridad que el error se debe a la ausencia de artículo (quizás por sobregeneralización, al suprimir el artículo en construcciones posesivas en las que debería aparecer porque no es nombre de familia en singular) y, en el ejemplo 3 , es el dominio imperfecto de los artículos el que da un resultado incorrecto (al contraer la preposición adecuada con un artículo que no lo es). La solución que hemos adoptado es que se considerará error preposicional cuando la elección, omisión o adición erróneas de artículo afecte a la preposición articulada, a la que tomamos como un único elemento lingüístico. Sin embargo, a la hora de hacer la valoración, tendremos en cuenta si la forma errónea se ha debido a problemas con la elección y uso de las preposiciones o con los artículos determinados.

A cada complemento empleado le hemos asignado un código (cuadro 9) para hacer más cómodo el recuento de los complementos más y menos utilizados y el porcentaje de error en cada uno de ellos (cuadros 10 y 11) $:$ AG = agente, $\mathrm{AR}=$ argomento, $\mathrm{C}=$ compagnia, $\mathrm{CA}=$ causa, $\mathrm{CO}=$ complemento oggetto, $\mathrm{D}=$ distributivo, $\mathrm{DI}=$ distanza, $\mathrm{E}=$ età, $\mathrm{F}=$ fine, $\mathrm{MAL}=$ moto a luogo, $\mathrm{MDL}=$ moto da luogo, $\mathrm{ME}=$ mezzo o strumento, $\mathrm{MO}=$ modo, $\mathrm{MPL}=$ moto per luogo, $\mathrm{O}=$ origine o provenienza, $\mathrm{PA}=$ paragone, $\mathrm{PAR}=$ partitivo, $\mathrm{RE}$ $=$ relazione, $\mathrm{S}=$ specificazione, $\mathrm{SIL}=$ stato in luogo, $\mathrm{T}=$ tempo, $\mathrm{TE}=$ termine, $\mathrm{U}=$ unione, $\mathrm{y}$ también se lo dimos a LA para la locuzione avverbiale, $\mathrm{LP}=$ locuzione preposiz̨ionale, $\mathrm{PRO}=$ proposizione (oggettiva, soggettiva, finale, etc.) y RA para la reggenza aggettivale.

\section{RESUltados}

Se ha registrado un total de 582 "usos preposicionales". Hemos de precisar que dicho número no se corresponde con el número total de preposiciones en el conjunto de las composiciones (que ha sido ligeramente inferior, 550). Veamos el porqué de esta diferencia. La valoración de uso preposicional correcto (426 casos) o de uso preposicional incorrecto (156 casos) -total 582- se ha hecho desde una doble perspectiva:

- italiana, en el sentido de que se ha valorado como uso correcto cuando en italiano el contexto o la expresión exigían una preposición y los alumnos elegían la adecuada (vado al cinema, vado molto d'accordo con mia sorella), tanto si dicha expresión en la L1 requería preposición (voy al cine) como si no (me llevo muy bien con mi hermana).

\footnotetext{
${ }^{6}$ En el apartado Anexos, en el cuadro número 8, figuran los ejemplos de las producciones idiosincrásicas de los aprendices con sus correspondientes versiones corregidas.

${ }^{7}$ El nombre de los complementos procede de la Grammatica italiana de M. Dardano y P. Trifone (1989).
} 
- española, valorando también como uso correcto cuando en italiano la expresión no requería preposición ( $\varnothing$ La mattina mi alzo presto, Domenica $\varnothing$ sera guardo la TV, Vedo spesso $\varnothing i$ miei amici), a pesar de que en la LM (español) equivalía a una expresión con preposición (Por la mañana me levanto temprano, El domingo por la tarde veo la TV, Veo a menudo a mis amigos), puesto que demostraba que no había tenido lugar una transferencia negativa del español al italiano y que el alumno, por tanto, había sabido diferenciar los usos preposicionales en ambos códigos lingüísticos.

Las preposiciones más usadas han sido: a (213 usos), di (127 usos), in (93 usos), y con (67 usos). Las menos usadas han sido: su y tra/fra que no han sido usadas ni una sola vez, da (18 usos) y per (32 usos). Si nos fijamos en los porcentajes ${ }^{8}$, la preposición con mayor porcentaje de acierto ha sido con $(100 \%)$, seguida de per $(87,50 \%)$ y a $(70,89 \%)$; mientras que las que han registrado mayor porcentaje de error han sido da $(50 \%)$, di $(38,58 \%)$ e in $(34,41 \%)$.

Los complementos más utilizados en respuestas correctas (cuadro 10) han sido: SIL (stato in luogo, 32,49\%), C / U (compagnia o unione, 15,23\%), MAL (moto a luogo, $13,71 \%$ ) y S (specificazione, $12,18 \%$ ) y los menos AR, PAR, PA, CA, MDL, AG, DI y D, con porcentajes inferiores al 1\%. Los complementos que, en cambio, han registrado más errores (cuadro 11) han sido: SIL (stato in luogo, 29,49\%), S (specificazione, 25,64\%), MAL (moto a luogo, 16,67\%), T (tempo, 8,33\%) y TE (termine, 5,13\%).

Veamos los resultados preposición por preposición, empezando por la más utilizada:

\subsection{La preposición $a$}

Ha sido utilizada 213 veces. Veamos cuáles han sido los valores con los que ha aparecido esta preposición, los casos en los que su empleo ha sido correcto y aquellos en los que se han producido errores?:

- en el complemento circunstancial de lugar en donde (stato in luogo, SIL) ha sido empleada 112 veces, en su mayoría bien -94 casos- y principalmente sin articular: abito a Saragozza, sono nato a León, rimango a casa, lavoro a Telepizza, studio qui a Zaragoza; sólo hay un caso con la preposición articulada studio inglese all'università. De los 18 casos incorrectos dentro de este valor, los problemas han sido los siguientes: falta de articulación entre la preposición y el artículo determinado: studio italiano a l'università, sono studentessa a la università di Saragozza, laboro a la discoteca Oasis; a en lugar de in: abito a Villasayas a Soria,

\footnotetext{
${ }^{8}$ Están recogidos en el apartado Anexos, cuadros numerados del 1 al 7.

${ }^{9}$ Los ejemplos que ofrecemos respetan el dialecto idiosincrásico (en palabras de Corder [1971] 1981: 14-25) de los aprendices; es decir, han sido transcritos tal y como lo hicieron los alumnos, con sus formas correctas y con aquellas que todavía no se adecuan a las de la lengua meta.
} 
abito a una residenza universitaria, lavora a una fabbrica; omisión del artículo determinado: sono al secondo anno di Diritto; adición de artículo: ceno a la mia casa (por a casa mia).

- en el complemento circunstancial de lugar adonde (moto a luogo, MAL) ha sido empleada 67 veces, bien en 41 casos y mal en los 26 restantes. De los 41 casos correctos, frente al valor anterior, los ejemplos con articulación de preposición y artículo determinado (casi exclusivamente is han superado a los de preposición simple (26 frente a 15): vado al mare, al bar, al cinema, andiamo a León, torno a casa, andiamo fuori a cena, vado a letto, andiamo spesso a teatro. De los 26 casos incorrectos, los problemas encontrados han sido: falta de articulación entre preposición y artículo: vado a l'università, a la facoltà, a la mia città, a la mia casa o articulaciones incorrectas: vado il fine di settimana ala mia piccola villa; a en lugar de in: abbiamo traslocato alla Germania, voglio andare a l'Italia, sono viaggiato a Francia, andare a Italia, vado a la disco, a la montagna, a la facoltà; omisión del artículo: mi piace andare a $\underline{\varnothing}$ cinema; adición de artículo: andare al letto; elección errónea de artículo: mi piace andare à le cinema, alle cinema, alle università.

- con valor temporal (T) ha sido empleada 11 veces, 5 bien y 6 mal. Los usos correctos eran indicaciones de horas en su mayoría con preposición articulada: mi alzo alle sette e mezza, le classe cominciano alle nove, la domenica mi alzo a mezzogiorno. De los usos incorrectos, los problemas han sido: falta de articulación: mi alzo a la sette, a le otto, a le nove et mezza y la adición de la preposición en expresiones temporales que no la precisan: quasi mai vado a la facoltà alla sera.

- introduciendo el complemento indirecto (complemento di termine, TE) ha sido empleada 4 veces y siempre correctamente -a pesar de la reduplicación (pronombre y forma nominal) del complemento-: a me mi piace, a tutti quattro $\underline{\underline{i}}$ piace, mi piace scrivere alle mie amiche.

- para indicar medio o instrumento (ME) ha sido empleada 3 veces, todas ellas para expresar la práctica de un juego o deporte, 2 bien: giocare a tennis, giocare a carte y una mal por la adición de artículo: giocare al calcio.

- dos casos de infinitivo precedido de preposición $a$, uno correcto: ho cominciato a studiare y otro incorrecto: vado à studiare.

- con adjetivos que rigen esta preposición (RA / LP) hemos encontrado 3 casos, siempre con el adjetivo vicino: 1 bien: vicina a Pamplona y 2 mal por falta de articulación: vicino a la montagna o por adición de artículo: vicino a la mia casa (por a casa mia).

- con valor distributivo (D) ha sido empleada 2 veces y correctamente: una volta alla settimana, una volta all'anno. 
- con valor modal (MO) ha sido empleada una sola vez y correctamente en la expresión al rovescio.

- en un único caso la preposición a ha sido utilizada en lugar de di para presumiblemente indicar origen $(\mathrm{O}): i$ miei genitori sono a León e abitano li.

- un caso de locución adverbial (LA) con a y erróneo por adición de artículo: al volte.

- se han cometido 3 errores de adición de la preposición a delante de complementos directos animados (CO): per visitare a mia madre, vedere agli amici, sempre bisogno a miei amici.

- se han cometido 3 errores de omisión de la preposición: delante del infinitivo imparemo [impariamo] $\varnothing$ scrivere, en complementos circunstanciales de lugar a donde (MAL) vado $\varrho$ l'università la mattina, quando ritorno $\varrho$ casa dell'università.

\subsection{La preposición $d i$}

Ha sido utilizada 127 veces. Veamos cuáles han sido los valores con los que ha aparecido esta preposición y examinemos, asimismo, los usos correctos y los que contienen errores:

- en el complemento de especificación (S) ha sido empleada 84 veces, 48 correctamente (mayoritariamente sin articular, 39 casos): grupo di musica, numero di telefono, corso di russo, professoressa di spagnolo, studentessa di Diritto, studio Storia dell'Arte, studentessa della facoltà di Lettere, cattedrale della Seo, Piazza del Pilar. En 36 casos se ha empleado incorrectamente debido a las siguientes razones: falta de articulación: nord est de la Spagna; articulación incorrecta: il nome dei miei grupo di musica, Storia del Arte, Storia del'Arte, Storia dell Arte; de ${ }^{10}$ en lugar de di: vedo film italiani de Luchino Visconti e de Fellini, una cosa che non mi piace de Saragozza è il troppo vento, secondo anno de Historia, studentessa de Filologia Inglese; omisión de la preposición: alle sette $\varrho$ sera, alle nove $\varrho$ mattina, studio secondo $\varnothing$ Diritto; adición

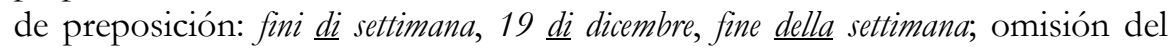
artículo: dintorni di mia città, studentessa di Facultad di Derecho, questa è la meior ora di giorno; adición de artículo: mio fratello fa il meccanico dell'aereo, vado a classe della lingua italiana; elección errónea de artículo: sono di un paese del_Pirinei aragonese.

- para la expresión de origen o procedencia $(\mathrm{O})$ ha sido utilizada 18 veces, en su mayoría correctamente: sono di Huesca, sono di León, mio padre è di Segovia. Sólo hay dos casos incorrectos debidos a falta de articulación: sono spagnola de la regione d'Aragón y di en lugar de da: quando ritorno a casa dell'università.

${ }^{10}$ Cf. Schmid (1994: 201), que explica el uso de en y de (por in y di respectivamente) por parte de aprendices hispanohablantes que se aproximan a la lengua meta a través de la estrategia de la congruencia. 
- con valor temporal ( $\mathrm{T}$ ) ha sido empleada 8 veces, 3 correctamente: d'estate, d'inverno y 5 incorrectamente en lugar de da: del lunedì al mercoledì, di lunedì a mercoledi, delle nove alle sette, sono sposata di un anno.

- con valor comparativo (PA) ha sido utilizada correctamente una vez: sono la più piccola di tutti y 5 veces ha aparecido che en lugar de di: $i$ miei fratelli sono più grandi hㅡio, ho un fratello più grande che io, la mia sorella è più piccola che io.

- con valor modal (MO) hemos encontrado 6 casos, todos en la expresión andare d'accordo y correctos.

- un único caso de di partitivo (PAR): scrivo delle lettere.

- un único caso introduciendo el complemento de argumento (AR): non bo ancora parlato della mia famiglia.

- ha aparecido en 3 locuciones (adverbiales o prepositivas LA/LP), dos correctas: di solito y una incorrecta: abito circa di la università (vicino all'università).

\subsection{La preposición in}

Ha sido utilizada 93 veces. Veamos cuáles han sido los valores con los que ha aparecido esta preposición, así como los casos en los que ha sido usada con corrección y en los que ha sido empleada erróneamente:

- en el complemento circunstancial de lugar en donde (stato in luogo, SIL), 34 casos correctos, principalmente sin articular: abita in Ingbilterra, sono nata in Germania, abito in periferia, si trova nel sud della Francia. De los 28 casos incorrectos, los problemas han sido: la falta de articulación: in la mia città, in la regione d'Aragón; in en lugar de $a$ : in Saragozza, in Teruel11, in Londra, studio in università, sono nel secondo anno di Storia, studio Geologia in l'università; en en lugar de in ${ }^{12}$ : en la mia famiglia, lavora en una farmacia, canto en un grupo; adición de artículo: è studiando in l'Irlanda, nel questo gruppo, in la Spagna, studiare in l'Italia.

- en el complemento circunstancial de lugar adonde (moto a luogo, MAL), 12 casos, todos correctos y con la preposición simple: vado in palestra, in discoteca, in facoltà.

- en el complemento circunstancial de tiempo (T), 13 casos, 10 correctos y principalmente sin articular: in inverno, in marzo, in questo momento, nel mio tempo libero y 3 incorrectos, bien por falta de articulación: in il mio tempo libero, bien por adición de preposición en contextos en los que no es necesaria: in la mattina vado in palestra, vado al cinema in domenica.

- en el complemento circunstancial de modo (MO), 2 casos, correctos y sin articular: essere in viaggio, vedo film in spagnolo.

${ }^{11}$ Se trata de las correspondientes ciudades (no provincias, que sí que irían introducidas por in).

12 Véase nota 10.

I.S.S.N. $1132-0265$

Philologia Hispalensis 21 (2007) 37-57 
- complemento de medio o instrumento (ME), 4 casos, 3 correctos: in bicicletta, in nave, nel treno y uno incorrecto por adición de artículo no necesario: voglio fare un viaggio nel treno.

\subsection{La preposición con}

Ha sido utilizada 67 veces y siempre correctamente. Veamos cuáles han sido los valores con los que ha aparecido esta preposición:

- el valor principal con el que ha sido usada esta preposición ha sido el de unión (U) o compañía (C) en 60 casos: mi piace andare al cinema con i miei amici, lavoro con bambini, mi piace uscire con gli amici.

- seguido del valor de relación (RE), siempre con la expresión andare d'accordo con qualcuno: con mio fratello vado molto d'accordo.

- en el complemento de medio o instrumento (ME) ha sido empleada una sola vez: lavoro con il ordinatore.

\subsection{La preposición per}

Ha sido utilizada 32 veces. Veamos cuáles han sido los valores con los que ha aparecido esta preposición, así como los usos correctos e incorrectos que hemos identificado:

- el valor principal ha sido el de interés o finalidad (F), con el que ha sido utilizada 18 veces y correctamente: lavoro come giornalista per un giornale per giovani, per me l'amicizia..., mi piace scrivere lettere per i miei amici, lavoro per guagnare soldi, mi piace viaggiare per conoscere nuove culture.

- en el complemento circunstancial de lugar por donde (moto per luogo, MPL) ha sido empleada 6 veces y correctamente: viaggiare per tutto il mondo, passeggiare per la città, andare in bicicletta per i dintorni.

- con valor temporal (T), 4 casos todos incorrectos por adición de preposición en partes del día: per la mattina, per la sera, leggo per la notte, o por omisión de la preposición: ho abitato qui $\underline{\varnothing}$ mesi13.

- en el complemento circunstancial de lugar adonde (moto a luogo, MAL) ha sido usada una sola vez y correctamente: partire per l'Italia.

- un caso con valor causal (CA) y correcto: per questo, ho un nome russo e un cognome tedesco.

- dos casos de LA (locurione avverbiale) y correctos: non mi piace per niente.

13 En la mayoría de las veces, para la expresión de tiempo continuado el significado es el mismo con per que sin la preposición (ho abitato li per tre mesi, ho abitato lì tre mesi). Sin embargo, cuando falta la indicación de medida, la preposición está siempre presente (bo abitato lì per mesi). El valor de per para la expresión de tiempo continuado es asimilado tarde por parte de aprendices hispanohablantes, debido probablemente a causas interlingüísticas. 


\subsection{La preposición $d a$}

Ha sido utilizada 19 veces. Veamos cuáles han sido los valores con los que ha aparecido esta preposición y, asimismo, los empleos correctos e incorrectos que hemos identificado respecto de esta preposición:

- en el complemento circunstancial de tiempo (T), 8 casos, 5 de ellos correctos y principalmente sin articular: abito a Zaragoza da molti anni, lavoro dalle 11 alle 8 , y 3 incorrectos por falta de artículo: abitiamo a Saragozza da $\varnothing$ 1991; omisión de la preposición: abitiamo qui $\underline{\varnothing}$ due anni; da en lugar de fa: [in California] dove noi ci abbiamo incontrato $\underline{d a} 5$ anni.

- en el complemento de separación, distancia o alejamiento (DI), un caso correcto y con preposición articulada: lontano dalla mia famiglia.

- en el complemento agente (AG) encontramos un único caso, correcto y sin articular: mia famiglia è costituita da quattro persone.

- en el complemento de origen o procedencia $(\mathrm{O})$, también un único caso y correcto: traduzione dallo spagnolo in russo.

- en el complemento de modo (MO) hemos encontrado un único caso, correcto y sin articular: stare da sola.

- hemos encontrado 4 casos en los que da ha sido utilizada incorrectamente en lugar de di en el complemento de especificación (S): sono studentessa da Filosofia,

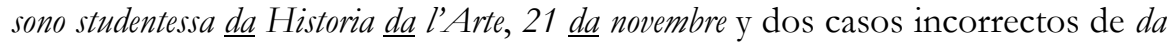
en lugar de $a$ en locuciones preposicionales: vicino da l'università, vicino da me.

\subsection{La preposición $s u$}

No ha sido utilizada ni una sola vez.

\subsection{La preposición tra/fra}

No ha sido utilizada ni una sola vez.

\section{CONCLUSiOnes}

Respecto de los resultados obtenidos, podemos extraer las siguientes conclusiones:

5.1. En primer lugar, consideramos que el total registrado de 582 usos preposicionales representa una importante presencia de preposiciones por composición (10-11). Los alumnos, aun sin conocer el paradigma preposicional italiano, ni los valores de cada preposición, ni las características particulares del sistema preposicional italiano, han hecho un uso considerable de esta categoría gramatical. Se podría objetar que no les quedaba otro remedio, porque de esta categoría es difícil prescindir, puesto que interviene prácticamente en todas las circunstancias de lugar, tiempo, modo, etc. 
5.2. El orden de las ocho preposiciones propias italianas, de mayor a menor presencia en las composiciones, ha sido el siguiente: $a$ (213 usos), di (127 usos), in (93 usos), con (67 usos), per (32 usos), da (18 usos), su y tra/fra (0 usos). El mayor o menor uso de cada preposición coincide, en cierto modo, con lo que sucede en su uso lingǘstico cotidiano ${ }^{14}$.

5.3. Siendo que el conocimiento de las preposiciones propias italianas hasta el momento de realizar esta prueba había sido inductivo, podemos afirmar que cuanto más rico sea el input que reciba el alumno, mejores serán sus producciones (output) -entendiendo como mejores, más ricas y más correctas-. El alumno se había presentado en clase, había oído a sus compañeros presentarse, se habían visto textos donde personajes diferentes hablaban de sî mismos o de sus familias y, por eso, se entiende que utilicen expresiones como andare d'accordo con qualcuno, que utilicen correctamente la preposición a en el complemento de lugar en donde (stato in luogo) en nada menos que 94 ocasiones: abito a Saragozza, lavoro a Telepizza, que hayan empleado correctamente la articulación entre la preposición y el artículo determinado en 54 ocasiones: studio inglese all'università, studio Storia dell'Arte, si trova nel sud della Francia, traduzione dallo spagnolo in russo, que la hayan utilizado -aunque con soluciones incorrectas- en otras 26 ocasiones: viaggiare al'Italia, Storia del'Arte, nel questo gruppo, y que hayan sabido omitir la preposición en contextos en los que aparecería en español: il pomeriggio guardo la tv, aiudare mia madre, dopo il pranzo.

5.4. El escaso o nulo uso de $s u$ (0 veces), tra/fra (0 veces), da (18 usos) puede deberse a la menor transparencia de significado de estas preposiciones para un alumno hispanohablante respecto de, por ejemplo, a, in, per, con, di. También podría deberse al tema de la composición, que pedía al alumno que hablase de sí mismo, de su familia, de sus gustos, etc., donde serían más requeridas preposiciones como di para explicar de dónde es uno, in y a para indicar circunstancias de lugar en donde o adonde (dónde viven, dónde trabajan, adónde les gusta ir en su tiempo libre), con para expresar complementos de compañía (con quién viven, con quién salen) que no $s u$ o tra/fra (indicaciones espaciales y temporales más concretas que tendrían mayor cabida en otro contexto lingüístico).

5.5 No sólo es interesante el número de veces que los alumnos sometidos a estudio han utilizado una preposición, sino las veces que han acertado utilizándola. La preposición $a$ se ha utilizado 213 veces, 151 veces bien (71\%)

\footnotetext{
14 Según el Lessico di frequenza dellitaliano parlato de T. De Mauro et al. (1993), el orden de las preposiciones propias italianas -comenzando por la más utilizada y terminando por la menos utilizada- es el siguiente: $d i, a$, in, per, da, con, su, tra/fra. En nuestro caso, sin embargo, se trata de un registro escrito.
} 
y $62 \mathrm{mal}(29 \%)$; di 127 veces, 78 bien (61\%), $49 \mathrm{mal}(39 \%)$; in 93 veces, 61 bien (66\%), $32 \mathrm{mal}(34 \%)$; con 67 veces, 67 bien (100\%); per 32 veces, 28 bien (87,5\%), $4 \mathrm{mal}$ (12,5\%); da 18 veces, 9 bien (50\%), $9 \mathrm{mal} \mathrm{(50 \% ).} \mathrm{Estos}$ porcentajes nos informan de que la preposición con no ofrece aparentemente problemas al aprendiz español de italiano y que las que más dificultad entrañan son $d a, d i$, in.

5.6. Los errores más comunes han sido los siguientes: la falta de articulación entre la preposición y el artículo determinado (Mi alzo a le sette ejemplo 1 , In la mia città ejemplo 2), articulaciones incorrectas (Mi alzo alla sette ejemplo 3, Studio Storia del Arte ejemplo 4), problemas en el dominio de los artículos determinados ${ }^{15}$ (Mi piace parlare con $\varnothing$ miei amici ejemplo 5, Abitiamo a Saragozza da 1991 ejemplo 6), confusión entre $a$ e in tanto en el stato in luogo-menoscomo en el moto a luogo -más- (Lavora a una fabrica ejemplo 7, Abito in Saragozza questo anno ejemplo 8, Voglio andare a l'Italia ejemplo 9, Vado a la disco il fine settimana ejemplo 10), confusiones entre da y di (Ho classe tre giomi del lunedì al mercoledì ejemplo 11, Sono studentessa da Filosofia e Letras ejemplo 12), adición y omisión de preposición (más frecuente ha sido el error de añadir preposición no necesaria -Il fine settimana vado al mio paese a visitare a mia madre ejemplo 13 , Per la mattina vado a la facoltà ejemplo 14- que el de omitirla cuando era precisa - Abitiamo qui $\underline{\varnothing}$ due anni ejemplo 15, Mi alzo alle sette $\varrho$ mattina ejemplo 16).

5.7. Las estrategias utilizadas por los aprendices en el campo de las preposiciones han sido varias. La transferencia de la lengua materna ${ }^{16}$ ha sido una de las principales: casos de en por in (En la mia famiglia ejemplo 17), de por di (Vedo

\footnotetext{
${ }^{15}$ La categoría del artículo en italiano presenta una problemática especial respecto del español, ya que el paradigma de los artículos en italiano es más amplio ( 7 en italiano -4 para el singular y 3 para el plural- en función no sólo del género y número de la palabra a la que acompañan sino también la letra por la que empieza esta palabra) y porque los contextos en los que el italiano requiere su presencia o la omite tampoco son los mismos que en español (el posesivo aparece con artículo -salvo en excepciones muy concretas- il mio cane, los años también lo requieren sono nata nel 1970, o los países -excepto en las circunstancias de moto a luogo o stato in luogo- Viene dall'Olanda; se omite, en cambio, en muchas expresiones de lugar andare a letto, in montagna, in discoteca, a teatro, in bagno). Los errores que afectan a esta categoría son tanto de naturaleza interlingüística (la influencia del español se deja notar sobre todo en el empleo del artículo en los mismos contextos que en español), como de naturaleza intralingüística (developmental error o error de desarrollo, cf. J. C. Richards (1971) en J. C. Richards ed. (1974: 172-188), que son producto de hipótesis equivocadas por parte del aprendiz sobre las reglas de la lengua meta, basadas en una experiencia limitada en la L2 o por el libro de texto.

16 S. Schmid (1994: 109-120) señala que la estrategia de la congruencia (descubrimiento de elementos y estructuras parecidos en distintos niveles del sistema entre la L1 y la L2, lo que hace que utilicen elementos de la L1 porque se suponen idénticos a los de la L2) es utilizada sobre todo en los primeros estadios de aprendizaje de una L2.
} 
film italiani de Fellini ejemplo 18), uso de la preposición a delante del OD animado (vedere agli amici ejemplo 19), traducciones literales del español (sempre bisogno a miei amici ejemplo 20, la mia sorella è più piccola che io ejemplo 21, abito circa di la università ejemplo 22), adición de preposición no necesaria reproduciendo expresiones que en español sí la requieren (per la notte leggo ejemplo 23), influencia de otras lenguas que conoce el aprendiz (el francés en el ejemplo 24 Mi piace andare à le cinema, el inglés en el ejemplo 25 quando ritorno $\varrho$ casa), hipercorrecciones (mio fratello fa il meccanico dell'aereo ejemplo 26, puesto que en español tampoco se emplearía el artículo), sobregeneralización de reglas de la lengua meta (como el caso de omitir la preposición en el moto a luogo en la frase vado Ø l'università la mattina ejemplo 27 , debido a la posible influencia que ejercía la omisión de preposición con una parte del día, regla que por otro lado el aprendiz podía conocer).

5.8. Otra estrategia que creemos ha sido empleada en las composiciones es la estrategia de la evitación o inbibición (avoidance) ${ }^{17}$. Se trata de una estrategia consciente a la que recurre el aprendiz de L2 y que consiste en no utilizar elementos o construcciones con los que no se encuentra cómodo o de los que no está seguro. Nos referimos, sobre todo, al hecho de que su y tra no hayan aparecido ni una sola vez. Fuera de la apreciación que hemos hecho anteriormente (5.4.), es posible que el alumno reconociese estos elementos como preposiciones en un texto y que fuese capaz de acercarse al significado pero, al tratarse de preposiciones más opacas (con poca similitud fonética con ninguna española) y con una frecuencia de aparición baja, ante estas circunstancias el alumno prefiere evitar su uso para asegurarse de que no comete error. De todos modos, la evitación es un fenómeno difícil de identificar y, para estar seguros de que ha tenido lugar, tendríamos que recurrir no a pruebas abiertas (como la composición) sino a otras de carácter más cerrado (tipo cloze, donde el alumno tuviese que elegir entre varias preposiciones incluyendo tra o $s u$ o ambas entre ellas) y saber que las conoce, porque el desconocimiento no es evitación (el aprendiz ha de saber lo que está evitando).

5.9. El hecho de que el aprendiz haya sabido utilizar las preposiciones correctamente en muchos contextos es algo muy positivo que no excluye en absoluto la presencia explícita de la gramática en clase. De hecho, pensamos que buena parte de los problemas que hemos detectado se solucionarían fácilmente y en muy poco tiempo haciendo expresas y practicando reglas gramaticales como la articulación de preposición y artículo determinado,

${ }^{17}$ El estudio clásico sobre este fenómeno es de J. Schachter (1974). Estamos de acuerdo con K. Smith (1982) en que esta estrategia -aunque no dé como resultado ningún error- aporta tanta información como los errores. 
ausencia de preposición $a$ delante del OD italiano. Del mismo modo, sería conveniente también que el profesor potenciase -o al menos no ignorase- la estrategia que los alumnos hispanohablantes emplean en su aprendizaje del italiano: nos referimos a la transferencia desde la LM que, aunque no siempre, en bastantes casos conduce a soluciones acertadas, explicando contrastivamente las diferencias de los paradigmas preposicionales español e italiano, los contrastes entre algunos pares de preposiciones que han demostrado ser más conflictivos insistiendo en que, en unos casos, en italiano la rección no es siempre de izquierda a derecha (como ocurre en la mayoría de los casos en español: ir a Madrid, a Italia, a la carnicería, a casa de María, a la montaña, a la cama, a la facultad; estar en Madrid, en Italia, en la carnicería, en casa de María, en la montaña, en la cama, en la facultad), sino que puede ser así en algunos casos (el origen se señala con di o con da dependiendo del verbo que preceda a la preposición essere di pero venire da) pero, en otros, es el elemento que va tras la preposición el que la escoge (andare/essere a Madrid, in Italia, in macelleria o dal macellaio, da Maria, in montagna, a letto, in facolta). Entre lenguas afines, es bastante lo que el alumno sabe (en principio, todo aquello que es igual o muy semejante), aunque él no esté muy seguro de que lo sabe porque no ha experimentado lo suficiente para conocer en qué casos es posible la transferencia. Las referencias a la LM del aprendiz por parte del profesor, indicando en qué casos por ejemplo la preposición a funciona como en español y en cuáles no, o que da equivale tanto a de, desde como a desde hace u ofreciendo abundantes ejemplos contextualizados en los que aparezcan los valores de las preposiciones más opacas ( $d a, s u$, tra/fra) puede ahorrar al alumno (y también al profesor) mucho tiempo y esfuerzo.

\section{REFERENCIAS BIBLIOGRÁFICAS}

ARCE, J, M. Carrera, F. Fernández Murga, Mª N. Muñiz, Italiano y español. Estudios lingüísticos, Sevilla, Publicaciones de la Universidad de Sevilla, 1984.

BAGNA, C. La competenza quasi-bilingüe/ quasi-nativa. Le preposiz̨oni in italiano L2, Milano, FrancoAngeli, 2004.

BERnINI, G. "Le preposizioni nell'italiano lingua seconda", Quaderni del Dipartimento di Linguistica e Letterature Comparate, Bergamo, Istituto Universitario, 1987, pp. 129152.

BosQue, I. y V. Demonte (eds.), Gramática Descriptiva de la Lengua Española, Madrid, Espasa Calpe, 1999.

CALVI, Ma V. Didattica di lingue affini. Spagnolo e italiano, Milano, Guerini. 1995.

CORDER, S. P. "The significance of learner's errors", International Review of Applied Linguistics, $\mathrm{n}^{\circ}$ 5, 1967, pp. 161-170.

CORDER, S. P. "Idiosyncratic dialects and error analysis", 1971, en Corder 1981, pp. $14-25$. 
CORDER, S. P. Introducing Applied Linguistics, Harmondsworth, Penguin Books Ltd., 1973.

CORDER, S. P. Error analysis and interlanguage, Oxford, Oxford University Press, 1981.

Dardano M. y P. Trifone, Grammatica italiana con nozioni di linguistica, Bologna, Zanichelli, 1989.

De MARCO A. (a cura di), Manuale di glottodidattica. Insegnare una lingua straniera, Roma, Carocci, 2000.

DE MAURo T. et al., Lessico di frequenza dellitaliano parlato, Milano, Etas Libri, 1993.

FAERCH C. y G. Kasper (eds.), Strategies in Interlanguage Communication, London, Longman, 1983.

FERNÁNDEZ López, C. Las preposiciones. Valores y usos, Salamanca, Ediciones del Colegio de España, 1999.

KATERINOV, K. La lengua italiana enseñada a estudiantes de habla española, Perugia, Guerra, 1979.

LARSEN-FreEman D. y M. H. Long, Introducción al estudio de la adquisición de segundas lenguas, Madrid, Gredos, 1994 (versión española de la versión original inglesa $A n$ Introduction to Second Language Acquisition Research, London, Longman, 1991).

MANCHÓN Ruiz, R. Ma "Estudios de interlengua: análisis de errores, estrategias de aprendizaje y estrategias de comunicación", Revista Española de Lingüistica Aplicada, $\mathrm{n}^{\mathrm{o}}$ 1, Madrid, SGEL, 1985, pp. 55-75.

OXFORD, R. L. Language Learning Strategies. What every teacher should know, Boston, Mass., Heinle \& Heinle Publishers, 1990.

PORRAS Castro, S. "Interferencia lingüística en la enseñanza/aprendizaje de una L2: español-italiano", Lenguaje y textos, Universidad de la Coruña, nº 4, 1991, pp. 67-72.

RiCHARDS, J. C. "A Non-Contrastive Approach to Error Analysis", English Language Teaching Journal, $\mathrm{n}^{\circ}$ 25, 3, 1971, pp. 204-19. Recogido en J. C. Richards (ed.), 1974, pp. 172-188.

Richards J. C. (ed.), Error Analysis. Perspectives on Second Language Acquisition, London, Longman, 1974.

Richards, J. C. J. Platt, H. Platt, Diccionario de lingüística aplicada y enseñanza de lenguas, Barcelona, Ariel, 1997 (versión española de C. Muñoz Lahoz y C. Pérez Vidal de la versión original inglesa: Longman Dictionary of Language Teaching and Applied Linguistics, London, Longman, 1992).

SCHACHTER, J. “An error in error analysis”, Language Learning, no 27, 1974, pp. 205-14.

SCHMID, S. L'italiano degli spagnoli. Interlingue di immigrati nella Svizzera tedesca, Milano, Franco Angeli, 1994.

SELINKER, L. "Language transfer", General Linguistics, no 9, 1969, pp. 67-92.

SELINKER, L. "Interlanguage", International Review of Applied Linguistics, n 10, 1972, pp. 209-231. 
SMITH, K "Avoidance, overuse and misure: three trial and error learning strategies of second language learners", Hispania, $\mathrm{n}^{\circ}$ 65, 1982, pp. 605-9.

Carmen Solsona Martínez

csolsona@,unizar.es

Departamento de Lingüística General e Hispánica

Facultad de Filosofía y Letras.

Universidad de Zaragoza

C/Pedro Cerbuna 12

50009 Zaragoza
Fecha de recepción: 11/01/2007

Fecha de aceptación: 3/07/2007 


\section{ANEXOS}

Cuadro 1 (Resumen de errores por usos preposicionales ${ }^{18}$

\begin{tabular}{|l|c|c|}
\hline Uso preposicional correcto & 426 & $73,20 \%$ \\
\hline Uso preposicional incorrecto & 156 & $26,80 \%$ \\
\hline Total & 582 & \multicolumn{1}{|l}{} \\
\cline { 1 - 2 } &
\end{tabular}

\section{Cuadro 2 (Preposición a)}

\begin{tabular}{|l|c|c|}
\hline Acierto & 151 & $70,89 \%$ \\
\hline Error & 62 & $29,11 \%$ \\
\hline
\end{tabular}

\section{Cuadro 3 (Preposición con)}

\begin{tabular}{|l|c|c|}
\hline Acierto & 67 & $100,00 \%$ \\
\hline Error & 0 & $0,00 \%$ \\
\hline
\end{tabular}

\section{Cuadro 4 (Preposición da)}

\begin{tabular}{|l|l|l|}
\hline Acierto & 9 & $50,00 \%$ \\
\hline Error & 9 & $50,00 \%$ \\
\hline
\end{tabular}

Cuadro 5 (Preposición di)

\begin{tabular}{|l|l|l|}
\hline Acierto & 78 & $61,42 \%$ \\
\hline Error & 49 & $38,58 \%$ \\
\hline
\end{tabular}

\section{Cuadro 6 (Preposición in)}

\begin{tabular}{|l|l|l|}
\hline Acierto & 61 & $65,59 \%$ \\
\hline Error & 32 & $34,41 \%$ \\
\hline
\end{tabular}

\section{Cuadro 7 (Preposición per)}

18 Nota: Su y tra/fra no han aparecido ni una sola vez. 


\begin{tabular}{|l|c|c|}
\hline Acierto & 28 & $87,50 \%$ \\
\hline Error & 4 & $12,50 \%$ \\
\hline
\end{tabular}

Cuadro 8 (Ejemplos de producciones idiosincrásicas)

\begin{tabular}{|l|l|}
\hline 1. Mi alzo a le sette & Mi alzo alle sette \\
\hline 2. In la mia città hanno molti monumenti & Nella mia città ci sono molti monumenti \\
\hline 3. Mi alzo alla sette & Mi alzo alle sette \\
\hline 4. Studio Storia del Arte & Studio Storia dell'Arte \\
\hline 5. Mi piace parlare con $\varnothing$ miei amici & Mi piace parlare con i miei amici \\
\hline 6. Abitiamo a Saragozza da 1991 & Abitiamo a Saragozza dal 1991 \\
\hline 7. Lavora a una fabrica & Lavora in una fabbrica \\
\hline 8. Abito in Saragozza questo anno & Abito a Saragozza quest'anno \\
\hline 9. Voglio andare a l'Italia & Voglio andare in Italia \\
\hline 10. Vado a la disco il fine settimana & Vado in discoteca il fine settimana \\
\hline 11. Ho classe tre giorni, del lunedì al mercoledì & Ho lezione tre giorni, dal lunedì al mercoledì \\
\hline 12. Sono studentessa da Filosofia e Letras & Sono studentessa di Lettere e Filosofia \\
\hline $\begin{array}{l}\text { 13. Il fine settimana vado al mio paese a } \\
\text { visitare a mia madre }\end{array}$ & $\begin{array}{l}\text { Il fine settimana vado al mio paese a visitare } \\
\text { mia madre }\end{array}$ \\
\hline 14. Per la mattina vado a la facoltà & La mattina vado in facoltà \\
\hline 15. Abitiamo qui due anni & Abitiamo qui da due anni \\
\hline 16. Mi alzo alle sette mattina & Mi alzo alle sette di mattina \\
\hline 17. En la mia famiglia siamo quatro & Nella mia famiglia siamo in quattro \\
\hline 18. Vedo film italiani de Fellini & Vedo film italiani di Fellini \\
\hline 19. Vedere agli amici & Vedere gli amici \\
\hline 20. Sempre bisogno a miei amici & Ho sempre bisogno dei miei amici \\
\hline 21. La mia sorella è più piccola che io & Mia sorella è più piccola di me \\
\hline 22. Abito circa di la università & Abito vicino all'università \\
\hline 23. Per la notte leggo un po & La notte leggo un po' \\
\hline 24. Mi piace andare à le cinema & Mi piace andare al cinema \\
\hline 25. Quando ritorno casa... & Quando ritorno a casa... \\
\hline 26. Mio fratello fa il meccanico dell'aereo & Mio fratello fa il meccanico di aerei \\
\hline 27. Vado l'università la mattina & Vado all'università la mattina \\
\hline
\end{tabular}




\section{Cuadro 9 (Códigos de complementos)}

\begin{tabular}{|c|c|}
\hline $\begin{array}{c}\text { Código } \\
\text { complemento }\end{array}$ & $\begin{array}{c}\text { Descripción } \\
\text { complemento }\end{array}$ \\
\hline
\end{tabular}

\begin{tabular}{|l|l|}
\hline- & nessuno \\
\hline AG & agente \\
\hline AR & argomento \\
\hline C & compagnia \\
\hline CA & causa \\
\hline CO & complemento oggetto \\
\hline D & distributivo \\
\hline DI & distanza \\
\hline E & età \\
\hline F & fine \\
\hline LA & locuzione avverbiale \\
\hline LP & locuzione preposizionale \\
\hline MAL & moto a luogo \\
\hline MDL & moto da luogo \\
\hline ME & mezzo o strumento \\
\hline MO & modo o maniera \\
\hline MPL & moto per luogo \\
\hline O & origine o provenienza \\
\hline
\end{tabular}

\begin{tabular}{|l|l|}
\hline PA & paragone \\
\hline PAR & partitivo \\
\hline PRO & proposizione (diversi tipi) \\
\hline RA & reggenza aggettivale \\
\hline RE & relazione \\
\hline S & specificazione \\
\hline SIL & stato in luogo \\
\hline T & tempo \\
\hline TD & tempo determinato \\
\hline TE & termine (OI) \\
\hline TF & tempo futuro \\
\hline TP & tempo passato \\
\hline U & unione \\
\hline X & complemento indecifrabile \\
\hline
\end{tabular}


Cuadro 10 (Porcentajes por usos correctos de complementos)

\begin{tabular}{|l|c|c|}
\hline SIL & 128 & $32,49 \%$ \\
\hline C / U & 60 & $15,23 \%$ \\
\hline MAL & 54 & $13,71 \%$ \\
\hline S & 48 & $12,18 \%$ \\
\hline F & 18 & $4,57 \%$ \\
\hline O & 16 & $4,06 \%$ \\
\hline T & 15 & $3,81 \%$ \\
\hline TE & 12 & $3,05 \%$ \\
\hline MO & 12 & $3,05 \%$ \\
\hline ME & 6 & $1,52 \%$ \\
\hline MPL & 6 & $1,52 \%$ \\
\hline RE & 6 & $1,52 \%$ \\
\hline D & 2 & $0,51 \%$ \\
\hline LA & 2 & $0,51 \%$ \\
\hline PRO & 1 & $0,25 \%$ \\
\hline RA & 1 & $0,25 \%$ \\
\hline DI & 1 & $0,25 \%$ \\
\hline PAS & 1 & $0,25 \%$ \\
\hline MDL & 1 & $0,25 \%$ \\
\hline CA & 1 & $0,25 \%$ \\
\hline PA & 1 & $0,25 \%$ \\
\hline PAR & 1 & $0,25 \%$ \\
\hline AR & 1 & $0,25 \%$ \\
\hline
\end{tabular}

Cuadro 11 (Porcentajes por usos incorrectos de complementos

\begin{tabular}{|l|c|c|}
\hline SIL & 46 & $29,49 \%$ \\
\hline S & 40 & $25,64 \%$ \\
\hline MAL & 26 & $16,67 \%$ \\
\hline T & 13 & $8,33 \%$ \\
\hline TE & 8 & $5,13 \%$ \\
\hline PA & 5 & $3,21 \%$ \\
\hline PRO & 4 & $2,56 \%$ \\
\hline CO & 3 & $1,92 \%$ \\
\hline O & 3 & $1,92 \%$ \\
\hline ME & 2 & $1,28 \%$ \\
\hline RA & 2 & $1,28 \%$ \\
\hline LA & 2 & $1,28 \%$ \\
\hline DI & 2 & $1,28 \%$ \\
\hline
\end{tabular}

